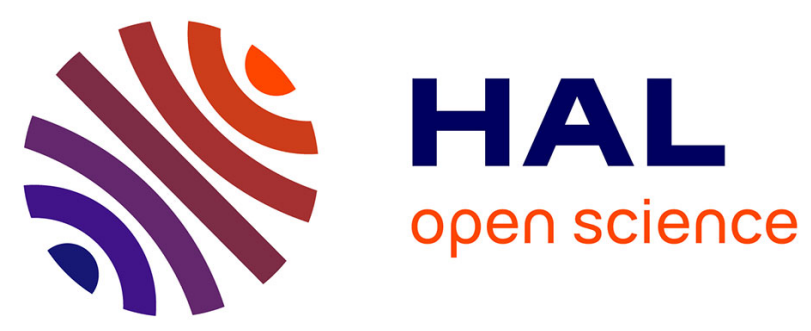

\title{
Nonlinear Electrostatic Steepening of Whistler Waves: The Guiding Factors and Dynamics in Inhomogeneous Systems
}

O. Agapitov, J. F. Drake, I. Vasko, F. Mozer, A. Artemyev, V. Krasnoselskikh, V. Angelopoulos, J. Wygant, G. D. Reeves

\section{To cite this version:}

O. Agapitov, J. F. Drake, I. Vasko, F. Mozer, A. Artemyev, et al.. Nonlinear Electrostatic Steepening of Whistler Waves: The Guiding Factors and Dynamics in Inhomogeneous Systems. Geophysical Research Letters, 2018, 45 (5), pp. 2168-2176. 10.1002/2017GL076957 . insu-01744922

\section{HAL Id: insu-01744922 \\ https://hal-insu.archives-ouvertes.fr/insu-01744922}

Submitted on 27 Mar 2018

HAL is a multi-disciplinary open access archive for the deposit and dissemination of scientific research documents, whether they are published or not. The documents may come from teaching and research institutions in France or abroad, or from public or private research centers.
L'archive ouverte pluridisciplinaire HAL, est destinée au dépôt et à la diffusion de documents scientifiques de niveau recherche, publiés ou non, émanant des établissements d'enseignement et de recherche français ou étrangers, des laboratoires publics ou privés. 


\section{Geophysical Research Letters}

\section{RESEARCH LETTER \\ 10.1002/2017GL076957 \\ Key Points: \\ - Nonlinear electrostatic steepening of whistler waves - the induced scattering to electron acoustic waves \\ - Resonant electron beam is highly modulated by a weakly oblique whistler wave \\ - Modulated electron beam initiates the electron acoustic wave turned into the electrostatic nonlinear mode phase related to the whistler}

Supporting Information:

- Supporting Information S1

Correspondence to:

O. Agapitov,

oleksiy.agapitov@gmail.com

\section{Citation:}

Agapitov, O., Drake, J. F., Vasko, I.,

Mozer, F. S., Artemyev, A.,

Krasnoselskikh, V., et al. (2018).

Nonlinear electrostatic steepening of

whistler waves: The guiding factors and dynamics in inhomogeneous systems. Geophysical Research Letters, 45. https:// doi.org/10.1002/2017GL076957

Received 4 JAN 2018

Accepted 23 FEB 2018

Accepted article online 1 MAR 2018

\section{Nonlinear Electrostatic Steepening of Whistler Waves: The Guiding Factors and Dynamics in Inhomogeneous Systems}

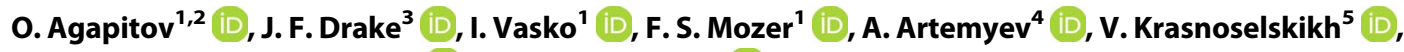 \\ V. Angelopoulos ${ }^{4}$, J. Wygant ${ }^{6}$ iD, and G. D. Reeves ${ }^{7}$
}

${ }^{1}$ Space Sciences Laboratory, University of California Berkeley, Berkeley, CA, USA, ${ }^{2}$ Astronomy and Space Physics Department, National Taras Shevchenko University of Kyiv, Kyiv, Ukraine, ${ }^{3}$ Department of Physics, the Institute for Physical Science and Technology and the Joint Space Institute, University of Maryland, College Park, MD, USA, ${ }^{4}$ Institute of Geophysics and Planetary Physics, University of California, Los Angeles, CA, USA, ${ }^{5}$ LPC2E/CNRS, Orleans, France, ${ }^{6}$ School of Physics and Astronomy, University of Minnesota, Twin Cities, Minneapolis, MN, USA, ${ }^{7}$ Space and Atmospheric Sciences Group, Los Alamos National Laboratory, Los Alamos, NM, USA

Abstract Whistler mode chorus waves are particularly important in outer radiation belt dynamics due to their key role in controlling the acceleration and scattering of electrons over a very wide energy range. The efficiency of wave-particle resonant interactions is defined by whistler wave properties which have been described by the approximation of plane linear waves propagating through the cold plasma of the inner magnetosphere. However, recent observations of extremely high-amplitude whistlers suggest the importance of nonlinear wave-particle interactions for the dynamics of the outer radiation belt. Oblique chorus waves observed in the inner magnetosphere often exhibit drastically nonsinusoidal (with significant power in the higher harmonics) waveforms of the parallel electric field, presumably due to the feedback from hot resonant electrons. We have considered the nature and properties of such nonlinear whistler waves observed by the Van Allen Probes and Time History of Events and Macroscale Interactions define during Substorms in the inner magnetosphere, and we show that the significant enhancement of the wave electrostatic component can result from whistler wave coupling with the beam-driven electrostatic mode through the resonant interaction with hot electron beams. Being modulated by a whistler wave, the electron beam generates a driven electrostatic mode significantly enhancing the parallel electric field of the initial whistler wave. We confirm this mechanism using a self-consistent particle-in-cell simulation. The nonlinear electrostatic component manifests properties of the beam-driven electron acoustic mode and can be responsible for effective electron acceleration in the inhomogeneous magnetic field.

Plain Language Summary We consider the effects of induced scattering of the electromagnetic whistler wave to the electrostatic electron acoustic wave (observed as field-aligned electric field bursts). The main discussed effect is based on the coupling of the slightly oblique whistler wave and a beam-driven electron acoustic wave observed as "nonlinear whistler waves". The wave interaction as the result produces the whistler wave and the rapidly steepening acoustic electrostatic wave with the same phase (and the same $k$ and frequency). Then, because the two different modes are the result of the interaction, the following dynamics of the waves in the inhomogeneous magnetic field is different: the whistler wave phase velocity depends on the background magnetic field magnitude but the acoustic mode propagate with the constant phase velocity. This dynamics leads to the waves phase differences and explains the fact that the observed in the experiment whistler and electrostatic bursts usually have actually random phase shift. To confirm this, we studied the dynamics of these waves in the inhomogeneous magnetic field system making use of the particle-in-cell simulation, reproduced all steps of the modes conversion, and confirmed that the dynamics in the inhomogeneous plasma system leads to the observed effects.

\section{Introduction}

Chorus whistler waves are important natural electromagnetic emissions in the magnetosphere because of their high efficiency in resonantly accelerating or precipitating electrons trapped in the outer radiation belt, producing either fast increases of high-energy (up to relativistic) electron fluxes or energetic electron precipitation. Observations by the Van Allen Probes (Mauk et al., 2013) provide high-quality extra low
C2018. American Geophysical Union. All Rights Reserved. 
frequency/very low frequency (VLF) measurements of the three wave electric field components (Wygant et al., 2012) including that parallel to the ambient magnetic field. These observations have revealed new evidence of rapid nonlinear wave-particle interactions in the outer radiation belt that can be important for electron acceleration and scattering on short time scales (see reviews by Albert et al., 2012, Artemyev et al., 2016, Omura et al., 2012, and Shklyar \& Matsumoto, 2009, and references therein).

The large parallel electric field components of oblique (relative to the background magnetic field) chorus waves allows them to interact with 0.1-1 keV electrons through the Landau resonance (Agapitov et al., 2015; Artemyev et al., 2013, 2016). Such electrons, trapped into the wave electrostatic potential, can provide nonlinear feedback that modifies the initial wave electric field, especially in the parallel direction, to produce the secondary harmonics reported by Kellogg et al. $(2010,2011)$ or the reported steepened parallel electric field bursts (Agapitov, Krasnoselskikh, et al., 2015; Drake et al., 2015; Mozer et al., 2015). However, the nature of these nonlinear whistler wave perturbations, as well as the favorable conditions for their development, has not been clear. The observed high amplitude (up to $200 \mathrm{mV} / \mathrm{m}$ ) very oblique whistlers (favorable for particle trapping in their effective potentials) have mostly exhibited monochromatic structures (Agapitov et al., 2014; Agapitov, Artemyev, et al., 2015; Cattell et al., 2008; Cully et al., 2008; Wilson et al., 2011). Clear nonlinear features were most evident in waves of intermediate amplitudes of $20-50 \mathrm{mV} / \mathrm{m}$ with only slightly oblique propagation (Agapitov, Mozer, et al., 2015; Drake et al., 2015; Kellogg et al., 2010; Mozer et al., 2015). Moreover, models of the nonlinear evolution of whistler waves suggested an amplitude threshold of around 0.1 of the background field (large compared to observed magnetospheric wave amplitudes) and/or the unusual wave propagation close to the resonance cone (Yoon et al., 2014). These facts indicate the need to study additional parameters controlling growth of higher harmonics, that is, steepening of whistler waves, as well as the nature and further dynamics of these nonlinear electric field perturbations.

In the following sections, we present new experimental features of nonlinear whistlers and provide the results of a 2-D particle-in-cell (PIC) simulation that reveals the development of nonlinear whistlers and their dynamics in an inhomogeneous magnetic field.

\section{Observational Properties of Nonlinear Whistler Steepening}

VLF and electron distribution function measurements from NASA's Van Allen Probes (Mauk et al., 2013) and Time History of Events and Macroscale Interactions define during Substorms (THEMIS) (Angelopoulos, 2008) were analyzed. We use the electric and magnetic field waveforms from the Electric Fields and Waves experiment (EFW) (Wygant et al., 2012) and the Electric and Magnetic Field Instrument Suite and Integrated Science (Kletzing et al., 2013) Van Allen Probes detectors and EFW (Bonnell et al., 2008) and SCM THEMIS detectors. The Van Allen Probe Flux Gate Magnetometer (Kletzing et al., 2013) and THEMIS Flux Gate Magnetometer (Auster et al., 2008) measurements were used to evaluate the background magnetic field. The electron distribution functions were obtained from the Energetic Particle, Composition, and Thermal Plasma suite (Spence et al., 2013) which included data from the Helium Oxygen Proton Electron detector for electron fluxes below 50 keV (Funsten et al., 2013) aboard Van Allen Probes and ESA (McFadden et al., 2008) aboard THEMIS.

The nonlinearity in whistler waveforms is observed predominantly in the parallel component of the electric field (Drake et al., 2015; Malaspina et al., 2014; Mozer et al., 2015). In Figures 1a and 1b, the transverse component of the wave magnetic field and the parallel component of the wave electric field captured aboard Van Allen Probe A on 24 April 2013 are presented. The spectrum of the magnetic field in Figure 1c shows the typical properties of the lower band chorus waves with a wave frequency of around $600 \mathrm{~Hz}$, or 0.35 of the local $f_{\text {ce. }}$. The electric field spectrum in Figure $1 \mathrm{~d}$ reveals significant harmonic generation up to $2 f_{\text {ce. }}$. This harmonic structure is clear from the normalized (to compare with the normalized magnetic field spectrum shown by the dashed curve) spectrum of parallel electric field in Figure 1e. The zoomed-in plots of electric and magnetic field waveforms show almost harmonic magnetic field variations (in Figure 1g) and periodic asymmetric bursts of parallel electric field in Figure 1f. The amplitude of the parallel electric field bursts exceed the wave parallel electric field amplitude calculated from the wave magnetic field amplitude by making use of the cold plasma whistler dispersion relation by about an order of magnitude (up to $40 \mathrm{mV} / \mathrm{m}$ compared to $2 \mathrm{mV} / \mathrm{m}$ expected from the whistler dispersion relation). The ratio of the wave magnetic field components indicates weakly oblique propagation with the wave normal angle around $20-30^{\circ}$ (relative to the background magnetic field). The crossphase behavior of the transverse magnetic and electric field components is in agreement with the whistler 


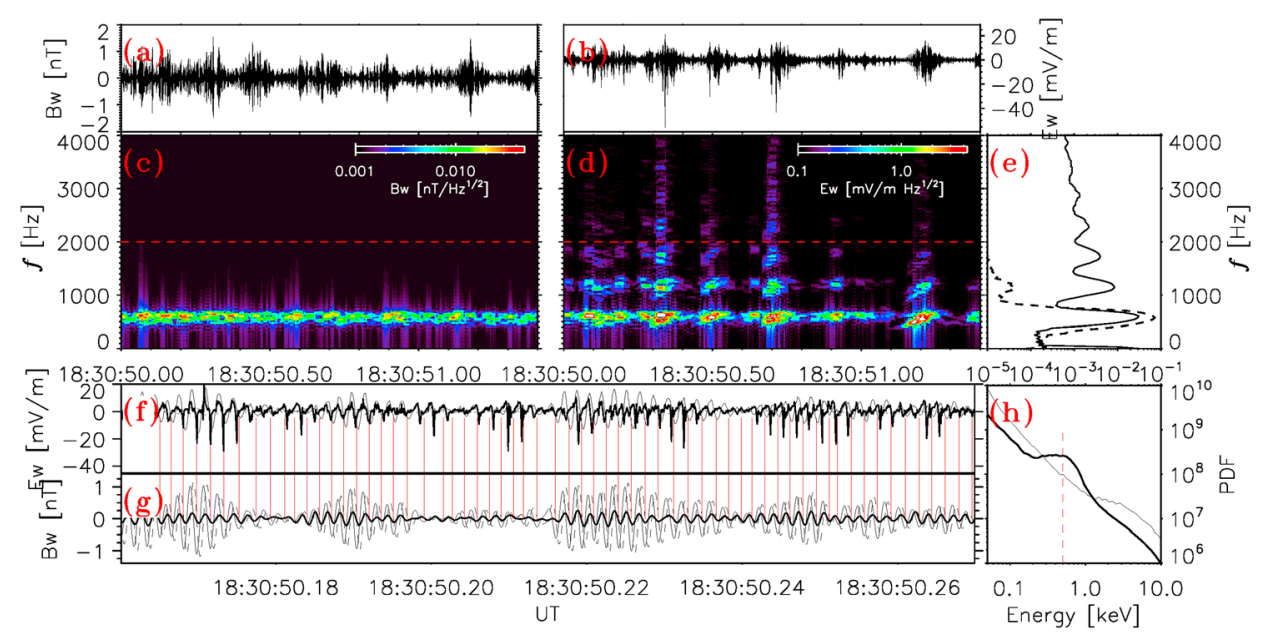

Figure 1. Magnetic and electric field waveforms $(a, b)$ captured aboard Van Allen Probe A on 24 April 2013 and their dynamic spectra $(c, d)$. The dashed red line represents the local electron gyrofrequency. Panel (e) presents the summary spectra of magnetic (the dashed curve) and parallel electric (the solid curve) field wave perturbation. The zoom of the wave perturbations is shown in panels $(\mathrm{g})$ and $(\mathrm{f})$ for the magnetic and electric field, respectively. In panel $(\mathrm{h}$ ) electron spectra along the magnetic field (the solid curve) and perpendicular to the field (the thin curve) are presented.

phase relationship. However, the maxima of the nonlinear parallel electric field bursts are not correlated with the whistler phase. Instead, they exhibit a nearly uniform distribution of the bursts versus the phases of either the transverse electric or magnetic field (as indicated by the vertical red lines). This indicates that there are random phase differences between the nonlinear parallel electric field perturbations and the whistler wave. This phase decoupling can take place only if the phase velocity of the electrostatic perturbation differs from the phase velocity of the whistler wave, that is, if two distinct plasma waves with close frequencies and different dispersion properties are observed propagating through the inhomogeneous plasma.

The electron distribution function in panel $(\mathrm{h})$ reveals a field-aligned beam-like anisotropy with a maximum that corresponds well with the phase velocity of the observed whistler wave. Thus, intense parallel electric field bursts can be amplified from oblique whistlers through interactions with electron beams (e.g., beamdriven electron acoustic mode, see Gary \& Tokar, 1985; Marsch, 1985). It is likely that the whistler wave parallel field modulates the electron beam and amplifies the beam at the wavelength scale. Such a modulated beam would drive electrostatic waves with a phase speed close to that of the whistler. As the beam propagates with a velocity close to the whistler wave phase velocity, the beam mode wave number is generated so that the electrostatic wave phase speed matches that of the whistler in the interaction region. Thus, the generated beam-driven mode has the same wave number as a whistler wave number. This scenario explains the oneto-one correlation between intense electrostatic parallel electric fields and whistler wave fields. Nonlinear interactions with electron beams (including particle trapping) should result in electrostatic wave steepening and formation of the observed electric field bursts. To test this scenario, we present a self-consistent numerical simulation of whistler wave generation and propagation together with electron beams that reveals the self-consistent generation of electrostatic waves with intense parallel electric fields. We also note that observed features (whistler wave, resonant electron beam, and nonlinear bursts of parallel electric fields) are not unique or exotic phenomena in the inner magnetosphere (see Figure S1 in the supporting information). The common feature of the observed cases is the relatively low phase velocities of the whistler waves due to the relatively high $\omega_{\mathrm{pe}} / \omega_{\mathrm{ce}}$ ratio (about 5-8), which satisfies the resonance condition of whistler and electron acoustic wave phase velocity equivalence.

\section{Computational Setup and Results}

We use a PIC simulation code similar to that used by (Drake et al., 2015) to generate enhanced fluctuations of both electromagnetic whistler and electrostatic electron acoustic waves. Our results are presented in normalized units: the magnetic field, predominantly directed along the $x$ axis, is normalized to $B_{0}$, time is in units of the electron cyclotron frequency $f_{\text {ce, }}$ lengths are relative to the electron skin depth $d_{\mathrm{e}}$, velocities are 
normalized to the electron Alfven speed $c_{\mathrm{Ae}}=B_{0} /\left(4 m_{\mathrm{e}} n_{0}\right)^{1 / 2}$ and temperatures to $m_{\mathrm{e}} c_{\mathrm{Ae}}{ }^{2}$. The computational domain is $L_{x} \times L_{y}=204.8 d_{\mathrm{e}} \times 204.8 d_{\mathrm{e}}$ with a grid scale of $0.05 d_{\mathrm{e}}$ (with 200 particles per cell). The ambient magnetic field magnitude $B_{0}$ depends on coordinates $x, y$ as $B_{0}=2+\cos \left(\pi \cdot x / 102.4 d_{\mathrm{e}}\right) \cdot \cos \left(\pi \cdot y / 102.4 d_{\mathrm{e}}\right)$, so $B_{0}$ varies by a factor of 3 along the $x$ direction at the center of the domain in $y$; the mass ratio is $m_{\mathrm{i}} / m_{\mathrm{e}}=200$, and the speed of light $c=5 c_{\mathrm{Ae}}$, while $\omega_{\mathrm{pe}} / \omega_{\mathrm{ce}}=5$. The main difference from the system setup in Drake et al. (2015) is the inhomogeneous magnetic field condition to reproduce better the conditions in the vicinity of the geomagnetic equator. The magnetic field inhomogeneity is the important factor for wave-particle interactions, which leads to wave/particle energy exchange (e.g., Shklyar \& Matsumoto, 2009). The simulations are carried out in a 2-D system with the initial magnetic field predominantly in the $x$ direction. The initial electron temperature is uniform $T_{\text {exx }}=T_{\text {eyy }}=T_{\text {ezz }}=0.025$, while, in the source region in the center domain, for $\left|x-L_{x}\right|<L_{x} / 12, T_{\text {eyy }}=T_{\text {ezz }}=0.4$, corresponding to the transverse anisotropy of 16 .

The whistler turbulence develops in the central $x$ region of the box, rapidly scattering the high-energy resonant electrons through the cyclotron resonance and decreasing their pitch angle to be more field aligned such that the anisotropy decreases by a factor of $\sim 5$ within a few tens of $1 / f_{\text {ce. }}$ The very high level of the initial anisotropy results in the rapid growth of whistler waves which scatter electrons nonlinearly (nondiffusively). This scattering leads to formation of structures in the particle distribution function, for example, a beam-like distribution of superthermal electrons propagating from the wave source. Formation of such structures cannot be described by quasi-linear diffusion and likely represent transient processes driven by the very strong anisotropy/rapid whistler wave growth. Although this beam generation involves parameters not observed under typical magnetosphere conditions, our model, which reproduces both oblique whistler waves and a resonant beam, successfully mimics spacecraft observations and thus can serve for investigation of the formation of strong electrostatic bursts. Whistlers propagate from the source as shown in the snapshot in the end of the simulation in Figure 2a (see also the supporting information to Drake et al., 2015). The typical magnetic field perturbations, shown in Figures $3 \mathrm{~b}$ and $3 \mathrm{e}$, have amplitudes up to $0.1 B_{0}$, which are about an orderof-magnitude larger than observed in the radiation belt. However, the nonlinear perturbations are not related to the maximum wave amplitude but are observed with $B_{w} \sim 0.03 \quad B_{0}$, which is comparable with the spacecraft observations in Figure 1 where $B_{w}$ is up to $1 \mathrm{nT}$ with $B_{0}=71 \mathrm{nT}$, that is, $B w \sim 0.15-0.2 B_{0}$. The ratio of the parallel to transverse components corresponds to wave normal angles around $20-30^{\circ}$, which is similar to the cases presented in Figures 1 and S1. In the following, we consider waves in the middle part of the system ( $y$ around 0 ), where whistlers are generated in a region with normalized $B_{0}=1$ and propagate to a region with a magnetic field of $B_{0} \sim 2-3$. Magnetic field lines are straight in this region so that the effects of magnetic field curvature are negligible. This region is intended to model the region in the vicinity of the geomagnetic equator in the Earth's outer radiation belt.

The relaxation of the very high electron temperature anisotropy during the generation of weakly oblique whistlers yields electron distributions with beam-like features along the direction of the ambient magnetic field (shown in Figures $2 \mathrm{~h}$ and $2 \mathrm{i}$ ). The beams propagate with velocities close to the quasi-parallel whistler wave phase speed. Therefore, our simulation setup reproduces the main observed conditions which are intense quasi-parallel whistlers that are Landau resonant with electron beams. The temporal modulation of the electron beam is evident in Figure $2 \mathrm{~d}$. Figure $2 \mathrm{c}$ (solid line) shows the electrostatic beam-driven mode. Nonlinear evolution of this electrostatic mode includes steepening (indicating the $\omega \sim k$ dispersion dependence), and the development of the nonlinear electrostatic bursts by electron trapping by potential wells (Figures $2 \mathrm{f}$ and $2 \mathrm{~g}$ ). The nonlinear structures are seeded by the whistlers through modulation of the electron beams propagating from the source region (due to the relaxation of the initial high transverse anisotropy). The power spectra of the wave electromagnetic components (presented in Figure $2 \mathrm{k}$ ) are nearly monochromatic with a wave frequency around $0.2 f_{\mathrm{ce}}$, and the higher harmonics in the spectrum of the electrostatic component of the wave contain significant energy, similar to the properties of the signal observed aboard the Van Allen Probes and THEMIS spacecraft (Figures 1 and S1). The harmonics keep a constant phase with respect to each other. The dispersion properties of the plasma waves observed in the spacecraft experiment (Figure 1) and in the PIC simulation (Figure 2) are presented in Figure 3. The quantities depicted in Figure 3 are described in the figure caption. The wavelength of whistler and electrostatic waves in the spacecraft data was estimated making use of the phase velocity and frequency value and was measured directly in the PIC results. The electrostatic harmonics propagate at a nearly constant velocity (all harmonics are coherent, i.e., keep the constant phase shift and propagate with the close velocity) indicating $k \sim \omega$, which is 

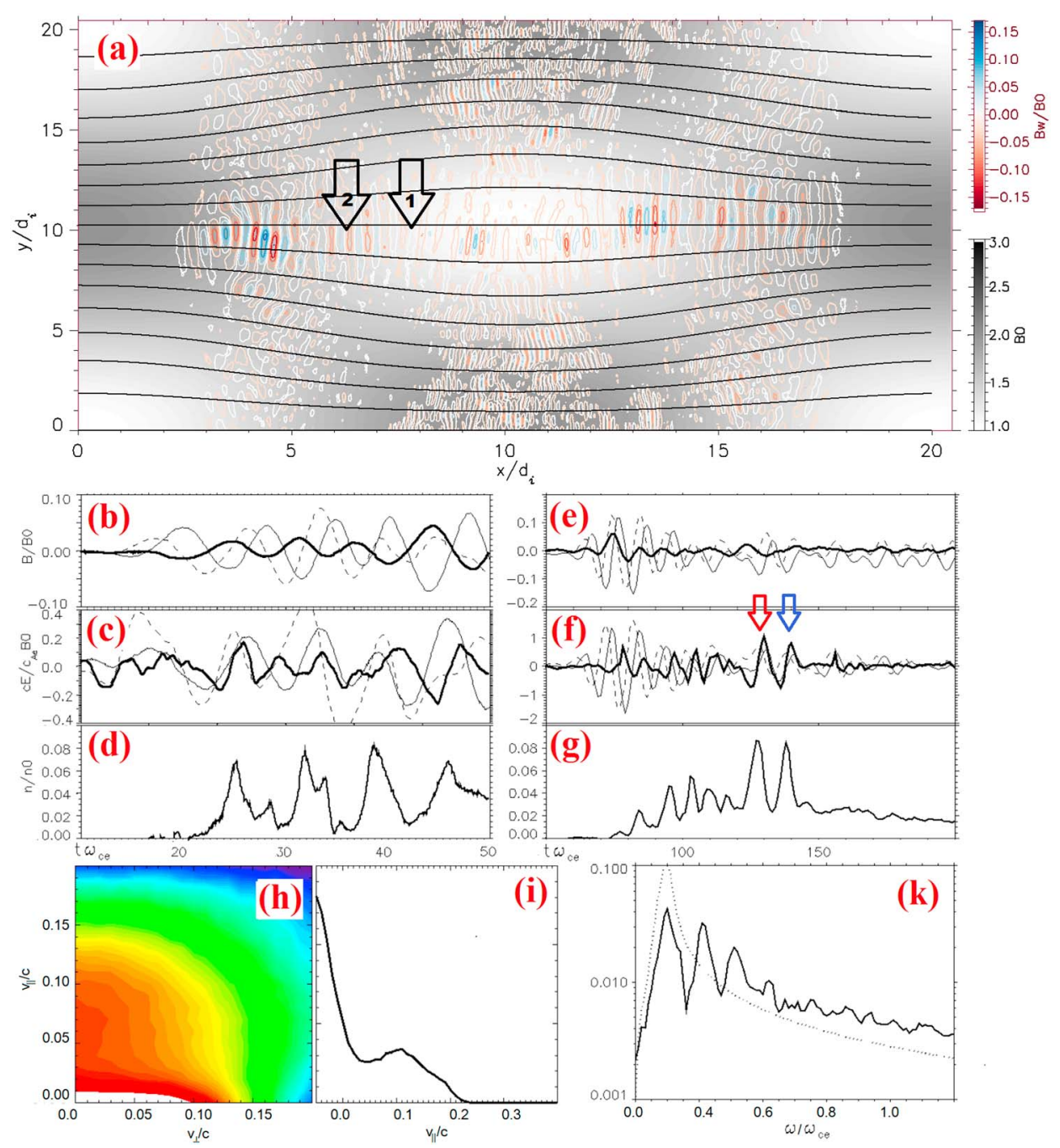

Figure 2. The system setup for the particle-in-cell run is presented in panel (a) with the magnetic field strength shown in the black/white palette. The red/blue contours show a snapshot of the whistler turbulence propagating from the source in the center of the domain. Panels (b) and (c) present the time-dependent magnetic and electric field perturbations collected at the point indicated by the black arrow \#1. Parallel components of the electric and magnetic field perturbation are indicated by the solid black curves. Panel (d) shows the modulation of the electron beam density as it propagates from the source region. In panels $(\mathrm{h})$ and (i) are the electron distribution function in the parallel and transverse velocity domain captured at point \#1 and the corresponding field-aligned electron distribution function (for $\left.v_{\perp}=0\right)$. Panels (e)-(g) present the magnetic and electric field perturbations as well as the beam density modulation captured at the point indicated at panel (a) by arrow\#2. The power spectrum of the parallel electric field perturbation is presented in panel $(k)$ with the solid black curve. Magnetic field spectrum is shown by the dashed curve.

significantly different from the whistler dispersion. The electrostatic bursts fall on a curve that corresponds to the dispersion of the beam-driven mode, so we conclude that they are the beam-driven electron acoustic waves. The train of periodic electrostatic bursts propagates at the same speed as the whistler wave. The amplitude of the electrostatic spikes appears to depend less on the initial whistler wave amplitude than on the beam flux: the nonlinear perturbations are not associated with the maximum amplitudes of whistler but with the maximum density of the beam. This explains the wide range of amplitudes of whistlers with nonlinear electrostatic components that are observed in the magnetospheric plasma. Electron acoustic waves are observed in the radiation belts in the presence of significant hot electron populations (Mozer et al., 2015; Vasko, Agapitov, Mozer, Bonnell, et al., 2017). Thus, these conditions might lead to similar nonlinear features. The electron acoustic wave is known to steepen rapidly (Dillard et al., 2018) and to propagate in a nonlinear mode in which trapping of resonant electrons in the wave potential takes place. 

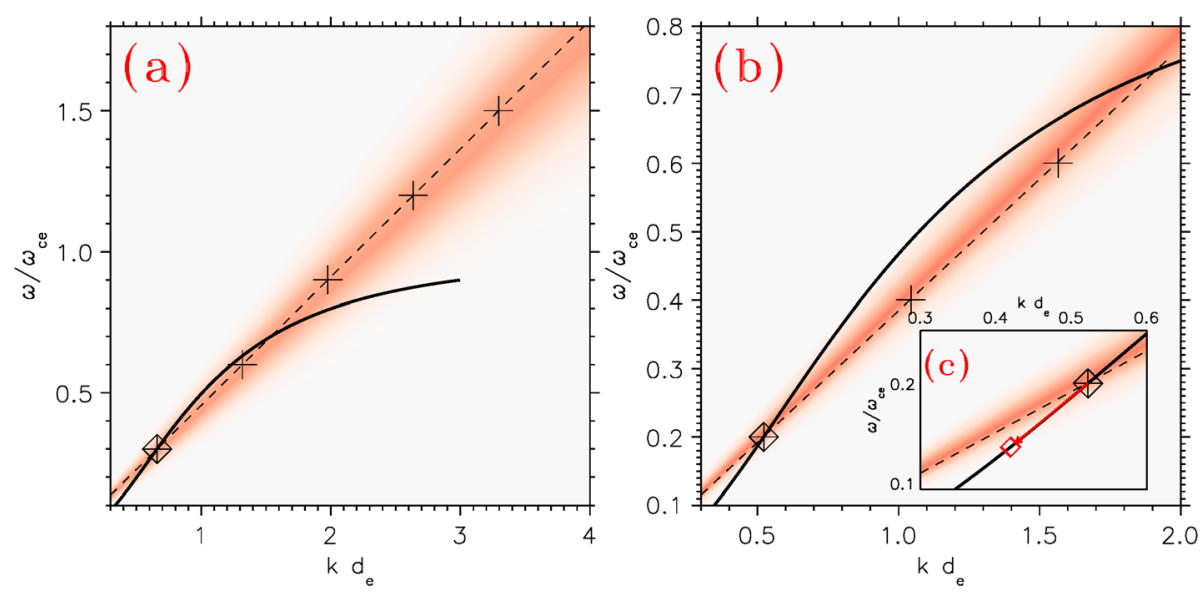

Figure 3. Panel (a) presents the dispersion relation of the whistler mode (the black solid curve) for the magnetic field and plasma parameters derived from the cold plasma linear approximation during the interval presented in Figure 1. The observed whistler wave (marked by the diamond) and the electrostatic wave harmonics (the cross signs) are shown. The positive slope velocity range from Figure $1 \mathrm{~h}$ is highlighted in red. The dashed line presents the electrostatic wave dispersion. Panel (b) presents the results of the particle-in-cell (PIC) simulation in the same format as panel (a): the whistler wave dispersion derived from the plasma parameters in the PIC setup and the electrostatic harmonics (the cross signs) obtained directly from the electric field perturbations. Panel (c) shows a zoom-in of panel (b), which emphasizes the vicinity of the wave coupling. The changes of the whistler frequency and wave number (small red diamond) due to wave propagation into the region with a larger background magnetic field, as observed in the PIC simulation, is highlighted by the red arrow.

Thus, linear damping might be suppressed (Montgomery et al., 2001; Nikolić et al., 2002). The nonlinear electron acoustic waves can play a significant role in wave-particle (Artemyev et al., 2014; Vasko, Agapitov, Mozer, Artemyev, et al., 2017) and wave-wave interactions (Agapitov, Krasnoselskikh, et al., 2015).

The phase velocity of whistlers depends on the ambient magnetic field magnitude (Stix, 1962) and increases along the $x$ direction as the waves propagate away from the source region in our simulation. Thus, the normalized whistler wave number changes during propagation and the coupling condition with the electron acoustic wave is lost, as illustrated in Figure $3 \mathrm{c}$. In Figure $4 \mathrm{~b}$, the space-time behavior of the wave magnetic
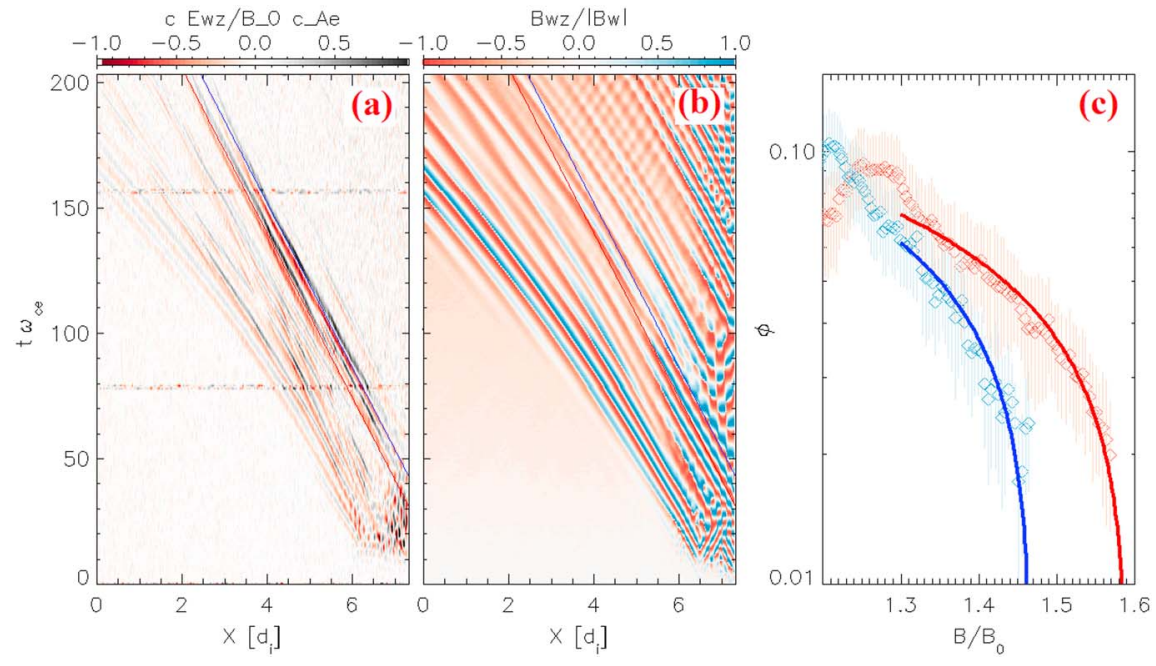

Figure 4. (a) Dynamics of the parallel electric field perturbation (averaged in the $9.5 d_{i}-9.9 d_{\mathrm{i}}$ range of the $y$ axis of the system shown in Figure 2a) presented in the space-time domain, where the $x$ axis represents the $x$ axis of the system in Figure $2 \mathrm{a}$ and the $y$ axis represents the time steps of the PIC simulation normalized to the source $\left(B_{0}=1\right)$ electron cyclotron frequency $\omega_{\text {ce }}$. The space-time behavior of the transverse magnetic field is shown in panel (b) in the same format. The red and blue curves in panels (a) and (b) indicate the propagation traces of the two nonlinear structures (marked in Figure $2 \mathrm{f}$ by the red and blue arrows). Panel (c) presents the amplitude of these two nonlinear structures as a function of the magnetic field strength (red and blue diamonds, respectively). The amplitude dynamics, computed from the local energy balance, are shown by the red and blue solid curves. 
field perturbation reveals an increase of the whistler phase and group velocity with distance $x$ from the source region. However, the beam-driven electrostatic waves should not depend on magnetic field since their velocity is dictated by the initial beam velocity. Indeed, Figure 4a shows that the parallel electric field bursts propagate with a constant velocity through the region with higher magnetic field. The traces of the two parallel electric field bursts indicated in Figure $2 \mathrm{f}$ by the arrows are shown in Figures $4 \mathrm{a}$ and $4 \mathrm{~b}$ by the red and blue curves. The difference in propagation velocities of the nonlinear electrostatic perturbations and the whistlers in the region of higher magnetic field demonstrates that the two waves become phase independent and explains the corresponding phase independence of the whistlers and electrostatic structures seen in the observations of the Van Allen Probes and THEMIS spacecraft.

The propagation of the electrostatic bursts containing trapped particles with constant beam velocity into the region with higher magnetic field magnitude leads to heating of the trapped population (an increase of the transverse temperature $T_{\mathrm{tr}}$ because of the conservation of the magnetic moment), which results in damping of these bursts seen in Figure 4c. The estimate of the damping from the energy exchange between trapped and untrapped populations gives a value of the peak electrostatic potential $\varphi$ that depends on the magnitude of the background magnetic field and predicts a decay of the amplitude of the structure as it moves through the region of increasing magnetic field along its trajectory. The amplitudes of the two bursts shown in Figure $4 \mathrm{a}$ in the red and blue trajectories are presented in Figure $4 \mathrm{c}$ (red diamonds for the first structure and blue diamonds for the second one) as a function of the ratio $B / B_{0}$. The rapid decay of the burst amplitude caused by the propagation into the region with the larger background magnetic field magnitude is in good agreement with the approximate dependence $e \varphi+T_{\mathrm{tr} 0} B / B_{0} \sim$ const with $T_{\mathrm{tr}}=T_{\mathrm{tr} 0} B / B_{0}$ shown by the red and blue solid curves, respectively.

\section{Discussion and Conclusions}

The Van Allen Probe and THEMIS satellites have recorded VLF waveforms of chorus-type whistler waves with significantly enhanced parallel electric field that exhibited sufficiently nonsinusoidal localized electrostatic bursts. The observed amplitudes of the parallel electric field exceed that expected from the ratio of $E_{w} / B_{w}$ estimated from the whistler dispersion relation in cold plasma by up to an order of magnitude. The power spectrum of the electrostatic perturbation has harmonic structure that is different from the whistler dispersion and contains significant wave power above $f_{\text {ce. }}$. The whistler waves identified in the observations were weakly oblique with wave normal angles of about $20-40^{\circ}$ and with modest amplitudes: the wave magnetic field was observed to be from 0.1 to $1 \mathrm{nT}$ and the wave electric field amplitude was about $10-20 \mathrm{mV} / \mathrm{m}$. This suggests that the thresholds for formation and development of electrostatic bursts synchronized with whistler waves do not require extreme whistler parameters but depend on the presence of an additional factor, which was found to be a resonant hot electron beam. The observed electrostatic bursts were shown to be likely nonlinear beam-driven electron acoustic waves with $\omega \sim k$ dispersion (Gary, 1985; Marsch, 1985; Tokar \& Gary, 1984), which are normally discounted in plasma systems due to their strong damping in the presence of a single electron component (Montgomery et al., 2001) but can be realized in the system having suppressed damping due to domination of the hot electron population or presence of the resonance electron beam. Coupling of the whistler mode with the electron acoustic mode (under conditions that make damping of the electron acoustic wave weak) provides the induced scattering of the whistler wave and determines the parameters of the resulting electrostatic wave.

We studied the generation and propagation of the nonlinear electrostatic perturbations stimulated by a weakly oblique whistler wave by making use a self-consistent numerical system using a 2-D PIC model as discussed in Drake et al. (2015). The strong anisotropy of the initial electron distribution and the strong resonant interaction of the growing whistler waves with the electrons result in formation of the system with whistler waves and an electron beam. This system mimics the observations and serves as a simulation model for investigation of the electrostatic wave growth and nonlinear dynamics. In the system with the resonant hot electron beam, the Landau damping of acoustic modes is suppressed. We observe the coupling of the electrostatic component of oblique whistlers with the electron acoustic mode, which drives the induced scattering through the modulation of the resonance beam and seeds the electrostatic electron acoustic wave generation. The electron acoustic wave rapidly steepens (due to $\omega \sim k$ dispersion) and grows into nonlinear electron acoustic structures (Drake et al., 2015). In the inhomogeneous magnetic field, the whistler phase 
velocity changes and the electrostatic bursts become phase independent from whistlers during propagation, as is also observed in the Van Allen Probes and THEMIS data.

Numerical simulations reveal an acceleration mechanism of electrons nonlinearly resonating with strong parallel electrostatic fields. This parallel electric field traps electrons, which are in resonance with the wave and the trapped electron population is efficiently heated as it is dragged to a higher ambient magnetic field. The particle gains transverse kinetic energy due to the conservation of the magnetic moment (this mechanism resembles the electron acceleration by quasi-electrostatic whistler waves, see, e.g., Artemyev et al., 2012, 2013; Agapitov et al., 2014; Agapitov, Artemyev, et al., 2015). The process results from the direct transformation of energy from electron beams to parallel electric field bursts and from these bursts to heating of the trapped electrons (with decrease of the pitch angles of the trapped population). As a result the parallel electric field bursts decay when propagating in the inhomogeneous magnetic field.

To conclude, in this paper we report the first direct observation of the electrostatic electron acoustic wave generation triggered by the electromagnetic whistler mode in the presence of the resonance electron beam. The process which has been thought of as the nonlinear electrostatic steepening of whistler waves is, instead, the coupling of the whistler with the electron acoustic mode. This plasma wave mode conversion is thought to be important for the energy transport to smaller scales and for plasma heating in space and laboratory plasma systems. This interpretation has been confirmed by a self-consistent numerical simulation that fully reproduces all steps of the process.

Acknowledgments

This work was supported by the JHU/APL contract 922613 (RBSP-EFW), NASA grant NNX16AF85G, NASA contract NAS5-02099, and NSF grants AGS1202330 and AGS1219369. V. K. acknowledges financial support of CNES through grant STEREO-WAVES invited scientist. Van Allen Probe EMFISIS data are at http://emfisis.physics.uiowa.edu/ data/index. EFW data are at http://www. space.umn.edu/rbspefw-data/. We acknowledge NASA contract NAS5-02099 and V. Angelopoulos for use of data from the THEMIS Mission: J. W. Bonnell and F. S. Mozer for use of EFI data; C. W. Carlson and J. P. McFadden for use of ESA data; $O$. LeContel, and the late A. Roux for use of SCM data. THEMIS data can be found at themis.igpp.ucla.edu.

\section{References}

Agapitov, O. V., Artemyev, A. V., Mourenas, D., Krasnoselskikh, V., Bonnell, J., Contel, O. L., et al. (2014). The quasi-electrostatic mode of chorus waves and electron nonlinear acceleration. Journal of Geophysical Research: Space Physics, 119, 1606-1626. https://doi.org/10.1002/ 2013JA019223

Agapitov, O. V., Artemyev, A. V., Mourenas, D., Mozer, F. S., \& Krasnoselskikh, V. (2015). Nonlinear local parallel acceleration of electrons through landau trapping by oblique whistler mode waves in the outer radiation belt. Geophysical Research Letters, 42, 10,140-10,149. https://doi.org/10.1002/2015GL066887

Agapitov, O. V., Krasnoselskikh, V., Mozer, F. S., Artemyev, A. V., \& Volokitin, A. S. (2015). Generation of nonlinear electric field bursts in the outer radiation belt through the parametric decay of whistler waves. Geophysical Research Letters, 42, 3715-3722. https://doi.org/10.1002/ 2015 GL064145

Agapitov, O. V., Mourenas, D., Artemyev, A. V., \& Mozer, F. S. (2016). Exclusion principle for very oblique and parallel lower band chorus waves Geophysical Research Letters, 43, 11,112-11,120. https://doi.org/10.1002/2016GL071250

Agapitov, O. V., Mozer, F. S., Artemyev, A. V., Mourenas, D., \& Krasnoselskikh, V. V. (2015). Wave-particle interactions in the outer radiation belts. ArXiv:1512.01863 [Physics]. Retrieved from http://arxiv.org/abs/1512.01863

Albert, J. M., Tao, X., \& Bortnik, J. (2012). Aspects of nonlinear wave-particle interactions. In D. Summers, et al. (Eds.), Dynamics of the Earth's radiation belts and inner magnetosphere (pp. 255-264). Washington, DC: American Geophysical Union. Retrieved from http://onlinelibrary. wiley.com/doi/10.1029/2012GM001324/summary

Angelopoulos, V. (2008). The THEMIS mission. Space Science Reviews, 141(1-4), 5. https://doi.org/10.1007/s11214-008-9336-1

Artemyev, A., Agapitov, O., Mourenas, D., Krasnoselskikh, V., Shastun, V., \& Mozer, F. (2016). Oblique whistler-mode waves in the Earth's inner magnetosphere: Energy distribution, origins, and role in Radiation Belt dynamics. Space Science Reviews, 200(1-4), 261-355. https://doi. org/10.1007/s11214-016-0252-5

Artemyev, A., Agapitov, O., Mozer, F., \& Krasnoselskikh, V. (2014). Thermal electron acceleration by localized bursts of electric field in the radiation belts. Geophysical Research Letters, 41, 5734-5739.

Artemyev, A., Krasnoselskikh, V., Agapitov, O., Mourenas, D., \& Rolland, G. (2012). Non-diffusive resonant acceleration of electrons in the radiation belts. Physics of Plasmas, 19(12), 122901.

Artemyev, A. V., Vasiliev, A. A., Mourenas, D., Agapitov, O. V., \& Krasnoselskikh, V. V. (2013). Nonlinear electron acceleration by oblique whistler waves: Landau resonance vs. cyclotron resonance. Physics of Plasmas, 20(12), 122901.

Auster, H. U., Glassmeier, K. H., Magnes, W., Aydogar, O., Baumjohann, W., Constantinescu, D., et al. (2008). The THEMIS fluxgate magnetometer. Space Science Reviews, 141(1-4), 235-264. https://doi.org/10.1007/s11214-008-9365-9

Bonnell, J. W., Mozer, F. S., Delory, G. T., Hull, A. J., Ergun, R. E., Cully, C. M., et al. (2008). The electric field instrument (EFI) for THEMIS. Space Science Reviews, 141(1-4), 303-341. https://doi.org/10.1007/s11214-008-9469-2

Cattell, C., Wygant, J. R., Goetz, K., Kersten, K., Kellogg, P. J., von Rosenvinge, T., et al. (2008). Discovery of very large amplitude whistler-mode waves in Earth's radiation belts. Geophysical Research Letters, 35, L01105. https://doi.org/10.1029/2007GL032009

Cully, C. M., Bonnell, J. W., \& Ergun, R. E. (2008). THEMIS observations of long-lived regions of large-amplitude whistler waves in the inner magnetosphere. Geophysical Research Letters, 35, L17S16. https://doi.org/10.1029/2008GL033643

Dillard, C. S., Vasko, I. Y., Mozer, F. S., Agapitov, O. V., \& Bonnell, J. W. (2018). Electron-acoustic solitary waves in the Earth's inner magnetosphere. Physics of Plasmas, 25(2), 022905. https://doi.org/10.1063/1.5007907

Drake, J., Agapitov, O., \& Mozer, F. (2015). The development of a bursty precipitation front with intense localized parallel electric fields driven by whistler waves. Geophysical Research Letters, 42, 2563-2570. https://doi.org/10.1002/2015GL063528

Funsten, H. O., Skoug, R. M., Guthrie, A. A., MacDonald, E. A., Baldonado, J. R., Harper, R. W., et al. (2013). Helium, oxygen, proton, and electron (HOPE) mass spectrometer for the Radiation Belt Storm Probes Mission. Space Science Reviews, 179, 423-484. https://doi.org/10.1007/ s11214-013-9968-7

Gary, P. S. (1985). Electrostatic instabilities in plasmas with two electron components. Journal of Geophysical Research, 90, 8213-8221. https:// doi.org/10.1029/JA090iA09p08213

Gary, S. P., \& Tokar, R. L. (1985). The electron-acoustic mode. The Physics of Fluids, 28(8), 2439-2441. https://doi.org/10.1063/1.865250 
Kellogg, P. J., Cattell, C. A., Goetz, K., Monson, S. J., \& Wilson, L. B. (2010). Electron trapping and charge transport by large amplitude whistlers. Geophysical Research Letters, 37, L20106. https://doi.org/10.1029/2010GL044845

Kellogg, P. J., Cattell, C. A., Goetz, K., Monson, S. J., \& Wilson, L. B. (2011). Large amplitude whistlers in the magnetosphere observed with wind-waves. Journal of Geophysical Research, 116, A09224. https://doi.org/10.1029/2010JA015919

Kletzing, C. A., Kurth, W. S., Acuna, M., MacDowall, R. J., Torbert, R. B., Averkamp, T., et al. (2013). The electric and magnetic field instrument suite and integrated science (EMFISIS) on RBSP. Space Science Reviews, 179(1-4), 127-181. https://doi.org/10.1007/s11214-013-9993-6

Malaspina, D. M., Andersson, L., Ergun, R. E., Wygant, J. R., Bonnell, J. W., Kletzing, C., et al. (2014). Nonlinear electric field structures in the inner magnetosphere. Geophysical Research Letters, 41, 5693-5701. https://doi.org/10.1002/2014GL061109

Marsch, E. (1985). Beam-driven electron acoustic waves upstream of the Earth's bow shock. Journal of Geophysical Research, 90, 6327-6336. https://doi.org/10.1029/JA090iA07p06327

Mauk, B. H., Fox, N. J., Kanekal, S. G., Kessel, R. L., Sibeck, D. G., \& Ukhorskiy, A. (2013). Science objectives and rationale for the Radiation Belt storm probes mission. Space Science Reviews, 179(1-4), 3-27. https://doi.org/10.1007/s11214-012-9908-y

McFadden, J. P., Carlson, C. W., Larson, D., Ludlam, M., Abiad, R., Elliott, B., et al. (2008). The THEMIS ESA plasma instrument and in-flight calibration. Space Science Reviews, 141(1-4), 277-302. https://doi.org/10.1007/s11214-008-9440-2

Montgomery, D. S., Focia, R. J., Rose, H. A., Russell, D. A., Cobble, J. A., Fernández, J. C., \& Johnson, R. P. (2001). Observation of stimulated electron-acoustic-wave scattering. Physical Review Letters, 87(15), 155001. https://doi.org/10.1103/PhysRevLett.87.155001

Mozer, F., Agapitov, O., Artemyev, A., Drake, J., Krasnoselskikh, V., Lejosne, S., \& Vasko, I. (2015). Time domain structures: What and where they are, what they do, and how they are made. Geophysical Research Letters, 42, 3627-3638. https://doi.org/10.1002/2015GL063946

Nikolić, L., Škorić, M. M., Ishiguro, S., \& Sato, T. (2002). Stimulated electron-acoustic-wave scattering in a laser plasma. Physical Review E, 66(3), 036404. https://doi.org/10.1103/PhysRevE.66.036404

Omura, Y., Nunn, D., \& Summers, D. (2012). Generation processes of whistler mode chorus emissions: Current status of nonlinear wave growth theory. In D. Summers, et al. (Eds.), Dynamics of the Earth's radiation belts and inner magnetosphere (pp. 243-254). Washington, DC: American Geophysical Union. Retrieved from http://onlinelibrary.wiley.com/doi/10.1029/2012GM001347/summary

Shklyar, D., \& Matsumoto, H. (2009). Oblique whistler-mode waves in the inhomogeneous magnetospheric plasma: Resonant interactions with energetic charged particles. Surveys in Geophysics, 30(2), 55-104. https://doi.org/10.1007/s10712-009-9061-7

Spence, H. E., Reeves, G. D., Baker, D. N., Blake, J. B., Bolton, M., Bourdarie, S., et al. (2013). Science goals and overview of the Radiation Belt storm probes (RBSP) energetic particle, composition, and thermal plasma (ECT) suite on NASA's van Allen probes mission. Space Science Reviews, 179, 311-336. https://doi.org/10.1007/s11214-013-0007-5

Stix, T. H. (1962). The theory of plasma waves. New York: AIP.

Tokar, R. L., \& Gary, S. P. (1984). Electrostatic hiss and the beam driven electron acoustic instability in the dayside polar cusp. Geophysical Research Letters, 11, 1180-1183. https://doi.org/10.1029/GL011i012p01180

Vasko, I. Y., Agapitov, O. V., Mozer, F. S., Artemyev, A. V., Krasnoselskikh, V. V., \& Bonnell, J. W. (2017). Diffusive scattering of electrons by electron holes around injection fronts. Journal of Geophysical Research: Space Physics, 122, 3163-3182. https://doi.org/10.1002/ 2016JA023337

Vasko, I. Y., Agapitov, O. V., Mozer, F. S., Bonnell, J. W., Artemyev, A. V., Krasnoselskikh, V. V., et al. (2017). Electron-acoustic solitons and double layers in the inner magnetosphere. Geophysical Research Letters, 44, 4575-4583. https://doi.org/10.1002/2017GL074026

Wilson, L. B., Cattell, C. A., Kellogg, P. J., Wygant, J. R., Goetz, K., Breneman, A., \& Kersten, K. (2011). The properties of large amplitude whistler mode waves in the magnetosphere: Propagation and relationship with geomagnetic activity. Geophysical Research Letters, 38, L17107. https://doi.org/10.1029/2011GL048671

Wygant, J., Bonnell, J., Goetz, K., Ergun, R., Mozer, F., Cattell, C., et al. (2012). Electric fields and waves instrument on the RBSP spacecraft: Investigating the dynamic of the inner magnetosphere. Abstract SM42B-08 Presented at the 2012 AGU Fall Meeting, San Francisco, CA.

Yoon, P. H., Pandey, V. S., \& Lee, D.-H. (2014). Oblique nonlinear whistler wave. Journal of Geophysical Research: Space Physics, 119, 1851-1862. https://doi.org/10.1002/2013JA018993 